\title{
Co-Precipitation of Trace Metals in Groundwater \& Vadose Zone Calcite: In Situ Containment \& Stabilization of Strontium-90 \& Other Divalent Metals \& Radionuclid
}

\author{
F. Grant Ferris \\ Department of Geology \\ University of Toronto \\ 22 Russell Street \\ Toronto, Ontario. M5S 3B1 \\ Canada
}

A suite of experiments were performed to investigate the partitioning of Sr2+ (to mimic the radionuclide 90Sr) between calcite and artificial groundwater in response to the hydrolysis of urea by Bacillus pasteurii under conditions that simulate in-situ aquifer conditions. Experiments were performed at 10, 15 and $20 \mathrm{C}$ over 7 days in microcosms inoculated with B. pasteurii ATCC 11859 and containing an artificial groundwater and urea (AGW), and an AGW including a Sr contaminant treatment. During the experiments ammonium concentration from bacterial urea hydrolysis increased asymptotically, and derived rate constants (kurea) that were between 13 and 10 times greater at $20 \mathrm{C}$, than at 15 and $10 \mathrm{C}$. Calcite precipitation was initiated after similar amounts of urea had been hydrolysed ( 4.0 mmoles L-1) and a similar critical saturation state (mean Scritical $=53$, variation $=20 \%$ ) had been reached, independent of temperature and $\mathrm{Sr}$ treatment. Because of the positive relationship between urea hydrolysis rate and temperature, precipitation began by the end of day 1 at $20 \mathrm{C}$, and between days 1 and 2 at 15 and $10 \mathrm{C}$. The rate of calcite precipitation increased with, and was fundamentally controlled by $\mathrm{S}$, irrespective of temperature, which connects the dissimilar patterns of urea hydrolysis and dissolved concentrations which are exhibited at the different experiments. The presence of Sr slightly slowed calcite precipitation rates at equivalent values of S, which may reflect the screening of active nucleation and crystal growth sites by Sr. Instantaneous heterogeneous partitioning coefficients (DSr) exhibited a positive association with calcite precipitation rates, but were greater at higher experimental temperatures at equivalent precipitation rates (20 C mean $=0.46 ; 15 \mathrm{C}$ mean $=0.24 ; 10 \mathrm{C}$ mean $=0.29$ ). This is likely to reflect the large ionic radius of the $\mathrm{Sr}$ ion, which cannot fully co-ordinate relative to ions smaller than $\mathrm{Ca}$ at equilibrium conditions, but is increasingly co-precipitated as all ions are indiscriminately incorporated at higher precipitation rates. The temperature dependence is likely to reflect the higher miscibility of ions in minerals, commonly observed in geochemical systems at higher temperatures. 